\title{
Conocimientos, habilidades y características del acceso a internet en estudiantes de medicina de una Universidad Peruana
}

\author{
PEDRO HORNA, WALTER CURIOSO, CARLOS GUILLÉN, CARLA TORRES, JORGE KAWANO \\ Facultad de Medicina Alberto Hurtado, Universidad Peruana Cayetano Heredia. \\ Sociedad Científica de Estudiantes de M edicina Cayetano H eredia - SOCEM CH.
}

\begin{abstract}
RESUMEN
OBJETIVOS: Estudiar los conocimientos, habilidades y características del acceso a internet en los estudiantes de medicina de pre-grado de una universidad peruana. MÉTODOS: Desarrollo y aplicación de una encuesta a estudiantes de medicina de una universidad peruana, reporte de resultados y estudio caso control anidado de variables relacionadas con la habilidad en internet. RESU LTA D OS: L os estudiantes accedieron a internet principalmente desde casa. $L$ as páginas más visitadas fueron las de correo electrónico y sitios de entretenimiento. Hubo una sobrevaloración de la calidad de información médica disponible en la red y, sin embargo, un pobre conocimiento de las herramientas de salud en internet, además de un deficiente dominio de algunas herramientas básicas de navegación y mensajería. El ser hábil en internet se relacionó significativamente con una larga experiencia en la red, la presencia de acceso a internet en casa y el uso frecuente del correo electrónico y la WW W. CONCLUSIÓN: L a población estudiada no posee las habilidades y conocimientos necesarios para aprovechar adecuadamente internet en su formación académica.
\end{abstract}

Palabras clave: Redes de comunicación de computadores; estudiantes de medicina; educación de pregrado en medicina; encuesta; países en desarrollo.

\section{MEDICAL STUDENTS' KNOWLEDGE, ABILITIESAND ACCESS CHARACTERISTICS TO THE INTERNET AT A PERUVIAN UNIVERSITY \\ ABSTRACT}

OBJECTIVES: To study the knowledge abilities and access characteristics to the Internet in undergraduate medical students from a Peruvian University. METHODS: Development and application of a questionnaire to medical students, report of the results and nested case-control analysis of data to identify variables related to the ability to surf the Internet. RESULTS: The students enter the Internet mainly from home. The most visited websites were those from web mail services and entertainment sites. The quality of the medical information available on the net was overrated, even though there was a poor knowledge of the online tools related to health, as well as a deficient ability with basic tools of navigation and e-mail. Being skillful on the Internet was significantly related to a long experience on the net, the presence of access to the Internet from home and the frequent use of e-mail and the WWW. CONCLUSIONS: The population studied does not have the ability and knowledge required to make the Internet a useful tool for its academic formation.

Key words: Computer communication networks; students, medical; education, medical, undergraduate; surveys; developing countries.

\section{Correspondencia:}

Pedro Horna Zegarra

Aurelio M iroquesada 154 D pto. 1701.

Lima 27, Perú

E-mail: pedrohorna@mail.com 


\section{INTRODUCCIÓN}

En los últimos 10 años, el crecimiento de internet como medio de comunicación masivo ha revolucionado el manejo e intercambio de información en medicina ${ }^{(1-3)}$. L a búsqueda automatizada, el acceso a literatura en formato electrónico y el intercambio de texto, imágenes y sonido en tiempo real ${ }^{4-6}$ ) son sólo al gunas de las características que hacen de internet un elemento imprescindible dentro de la práctica médica ( $\left.{ }^{7}\right)$ y los actuales estándares de la educación médica $\left(^{8}\right)$.

En países en desarrollo, muchas facultades de medicina han implementado cambios en la currícula e infraestructura universitaria, en un intento por integrar a internet dentro de sus actividades académicas, a pesar de las limitaciones inherentes a la situación económica de estas naciones $\left({ }^{9,10}\right)$. L amentablemente, no existen reportes previos acerca de los conocimientos, habilidades y características del acceso a internet entre los estudiantes de medicina del tercer mundo, que permitan contemplar estrategias inteligentes con recursos limitados.

En este sentido, nosotros reportamos los resultados de una encuesta realizada durante el mes de octubre de 1999 en estudiantes de medicina de una universidad privada ubicada en la capital peruana de Lima, con el fin de estudiar las características de esta población en cuanto al concepto que se tiene de internet, la capacidad percibida por los estudiantes para realizar tareas en la red, la frecuencia y características del acceso y los factores relacionados a un manejo adecuado.

\section{MATERIALES Y MÉTODOS}

Se estudió una población de 690 estudiantes de medicina matriculados en la U niversidad Peruana $\mathrm{C}$ ayetano $\mathrm{H}$ eredia, entre el primer a sexto año de estudios, excluyendo a los autores. En base al listado de alumnos proporcionado por la Oficina de Pre-Grado se obtuvo una muestra probabilística, aleatoria y estratificada por año de estudios de 275 estudiantes, de los cuales 227 respondieron la encuesta (tasa de rechazo, $17,5 \%)$.

Todos los estudiantes recibieron el mismo cuestionario, desarrollado sobre la base a una revisión bibliográfica y nuestra experiencia como estudiantes de medicina y miembros de un comité estudiantil de promoción de internet. Se realizó una encuesta piloto a inicios de setiembre en una muestra casual de 28 estudiantes del sétimo y octavo año medicina y se modificó el cuestionario de acuerdo a los resultados.

Durante las dos primeras semanas de octubre de 1999, un equipo de 10 colaboradores (estudiantes de medicina no seleccionados en la muestra) ubicó a los sujetos seleccionados durante actividades dentro de la universidad y, luego de explicarles la relevancia del estudio, les entregó la encuesta para ser devuelta el mismo día en una segunda visita.

Con fines ilustrativos, las proporciones de las variables categóricas fueron reportadas excluyendo las respuestas en blanco, cuando estas últimas representaban menos del $5 \%$ del número de sujetos en los cuales la pregunta era aplicable. EI intervalo de confianza del 95\% (IC 95\%) para las proporciones fue calculado considerando una distribución normal de $p$ y un tamaño poblacional de 690 estudiantes entre $1^{\text {er }}$ y $6^{\circ}$ año.

Los estudiantes que respondieron la encuesta fueron clasificados arbitrariamente como hábiles o no hábiles en internet, sobre la base a un puntaje igual al número de respuestas afirmativas a 10 preguntas acerca de tareas comunes de mensajería y navegación en internet (Tabla 1), a las cuales los sujetos debían afirmar o negar si podían realizarlas correctamente y sin asistencia. Los sujetos que obtuvieron un puntaje mayor a 4 (por encima del percentil 25) fueron clasificados como hábiles, mientras que aquellos cuyo puntaje era menor o igual a 4 ó que marcaron nunca haber ingresado a internet (puntaje $=0$ ) fueron clasificados como no hábiles. Se excluyó 7 sujetos $(3,1 \%)$ con respuestas en blanco a alguna de las 10 preguntas. 
Tabla 1. Porcentaje de estudiantes que afi rmaron poder real izar correctamente y sin asistencia distintas tareas en internet.

\author{
Tareas de navegación en la W W W \\ Entrar a una página conociendo la dirección web \\ Usar un buscador para encontrar información en internet (ej. Yahoo!) \\ Grabar el texto de una página web en un disquete \\ Grabar una imagen de una página web en un disquete \\ Obtener documentos de internet (ej. en Word) y abrirlos en la computadora \\ Obtener programas de internet e instal arlos en la computadora
}

Respuestas afirmativas ${ }^{1}$

$89,7 \% \pm 3,3 \%$

$88,4 \% \pm 3,5 \%$

$72,3 \% \pm 4,8 \%$

$55,6 \% \pm 5,4 \%$

$55,6 \% \pm 5,4 \%$

$35,7 \% \pm 5,2 \%$

$81,1 \% \pm 5,2 \%$

$61,0 \% \pm 5,2 \%$

$59,9 \% \pm 5,3 \%$

$51,6 \% \pm 5,4 \%$

${ }^{1}$ I ntervalo de confianza del 95\% para la población de 690 estudiantes de medicina.

Se realizó un análisis de caso-control anidado, para determinar diferencias significativas entre los sujetos hábiles y no hábiles en internet, con respecto a las demás variables. L as edades fueron comparadas con la prueba de t de Student, las variables nominales con la prueba de $\mathrm{Chi}$ cuadrado y las variables ordinales y las frecuencias con la prueba de M ann W hitney. Se calculó el "odds ratio" (OR) y el intervalo de confianza del $95 \%$ para las variables dicotómicas.

\section{RESULTADOS}

De los 227 estudiantes que respondieron a la encuesta, $68,3 \%$ eran varones y la edad promedio fue 21,5 años (desviación estándar $\pm 2,23$ años). Casi todos $(98,2 \%)$ habían accedido alguna vez a internet y 95,1\% lo había hecho en el último trimestre.

El $48,7 \%$ de los encuestados (IC 95\%, 42$55 \%$ ) afirmó tener conexión a internet en casa, la gran mayoría de los cuales $(91,7 \%)$ había usado esta vía como el principal medio de conexión a internet durante el último trimestre. De los estudiantes sin conexión en casa, 59\% declaró utilizar las computadoras de la universidad como el principal medio de acceso a internet, mien- tras que $23,8 \%$ declaró acceder principalmente desde cabinas públicas y un menor porcentaje desde otros lugares. EI 56,9\% de los sujetos sin conexión en casa manifestó tener una computadora.

En cuanto a la experiencia en internet y en computadoras, la mayoría de estudiantes se autocalificó como alguien que usa ocasionalmente estas facilidades o cuyos conocimientos se limitan a unas cuantas herramientas (Figura 1 ). En la Tabla 1 se resume los resultados de las preguntas acerca de la capacidad para realizar tareas específicas de navegación y mensajería en internet.

El $75,1 \%$ de los estudiantes (IC 95\%, 70,5$79,7 \%$ ) identificó la mejor definición de internet enunciada como "una red internacional de computadoras interconectadas que difunden e intercambian información", entre otras 4 definiciones erradas enunciadas como "una gran computadora central..." (8,4\%), "una compañía transnacional proveedora de información..." $(6,2 \%)$ y "una colección de discos compactos..." $(2,7 \%)$. Un $4,4 \%$ marcó que no estaba seguro cómo funcionaba internet y $3,1 \%$ consideró que ninguna de las alternativas era satisfactoria. $L$ a Tabla 2 muestra los resultados de las preguntas 


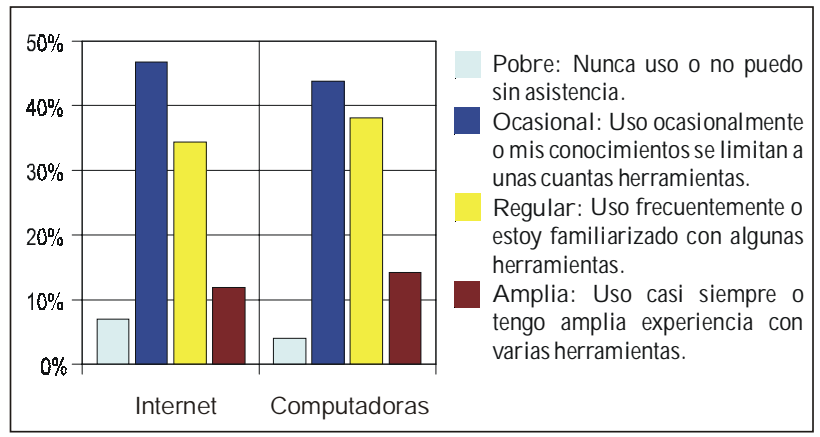

Las barras representan el porcentaje de sujetos que marcaron cada una de las cuatro al ternativas.

Figura 1. Autoapreciación de la experiencia en el uso de internet y en el uso de computadoras.

acerca de la percepción de la calidad de información médica disponible en internet.

El 91,4\% de encuestados declaró haber accedido a la "World Wide Web" (WW W) en el último trimestre, de los cuales sólo $11,4 \%$ marcó haber ingresado principalmente a páginas relacionadas a ciencias de la salud. En el resto, las páginas más visitadas fueron las de correo electrónico en la web $(44,8 \%)$, entretenimiento $(33,8 \%)$ u otra clase de páginas $(4,5 \%)$, mientras que $5,5 \%$ no marcó alguna alternativa. $L a$ frecuencia de acceso a la WW W en este periodo se muestra en la Figura 2.

Casi todos los estudiantes $(94,6 \%)$ reportaron haber ingresado alguna vez a la WWW en busca de información médica. Sin embargo, sólo algunos afirmaron haber encontrado el ementos básicos de información médica en la red, tales como bases de datos con resúmenes de publicaciones ("abstracts") (77,6\%), información sobre grandes centros médicos del mundo $(56,2 \%)$, publicaciones confiables de medicina en texto completo $(45,2 \%)$ y manuales, guías o consensos de tópicos médicos $(42,9 \%)$.

A penas tres sujetos $(1,4 \%)$ reportaron no haber encontrado problemas al buscar información médica en la red. El resto declaró tener problemas debido a fallas o lentitud en la conexión $(62,4 \%)$, desconocimiento de direcciones web $(61,4 \%)$, costo de conexión $(43,8 \%)$, falta de dominio de herramientas de internet $(40 \%)$, falta de dominio del idioma inglés $(22,9 \%)$, falta de dominio general de computadoras $(16,2 \%)$ y otros $(3,3 \%)$.

El sistema de correo electrónico más popular fue los correos gratuitos en la WW W, utilizados por $56,1 \%$ de los estudiantes encuestados durante el último trimestre. En este periodo sólo 24, 9\% reportó haber accedido al correo el ectrónico proporcionado por la universidad y $28,5 \%$ al correo de su proveedor de acceso a internet. Tres sujetos $(1,4 \%)$ declararon haber utilizado otra modalidad de correo electrónico. La frecuencia de acceso a cualquiera de estos correos durante el último trimestre se muestra en la Figura 3.

A I preguntar a los estudiantes que alguna vez habían accedido a internet, el tiempo transcurrido desde que tuvieron el primer contacto con

Tabla 2. Percepción de la cal idad de la información médi ca disponi ble en internet.

\begin{tabular}{|c|c|c|c|}
\hline \multicolumn{2}{|c|}{$\begin{array}{l}\text { ¿Que clase de información crees que es posi ble en- } \\
\text { contrar gratuitamente en internet acerca de la ma- } \\
\text { yoría detópicos médicos especial izados? }\end{array}$} & \multicolumn{2}{|c|}{$\begin{array}{l}\text { ¿Qué proporción de la información médica en } \\
\text { internet crees que provienede unafuente confiable? } \\
\text { (Puede ser citada en una revista, libro o tesis) }\end{array}$} \\
\hline Específica y extensamente desarroll lada & $30,2 \%$ & Toda & $5,0 \%$ \\
\hline Específica, pero poco desarrollada & 49,5 & Lama & $53,8 \%$ \\
\hline Muy general & $18,9 \%$ & Sólo una parte & $39,8 \%$ \\
\hline No es posible encontrar esta información & $1,4 \%$ & Nada & $1,4 \%$ \\
\hline
\end{tabular}




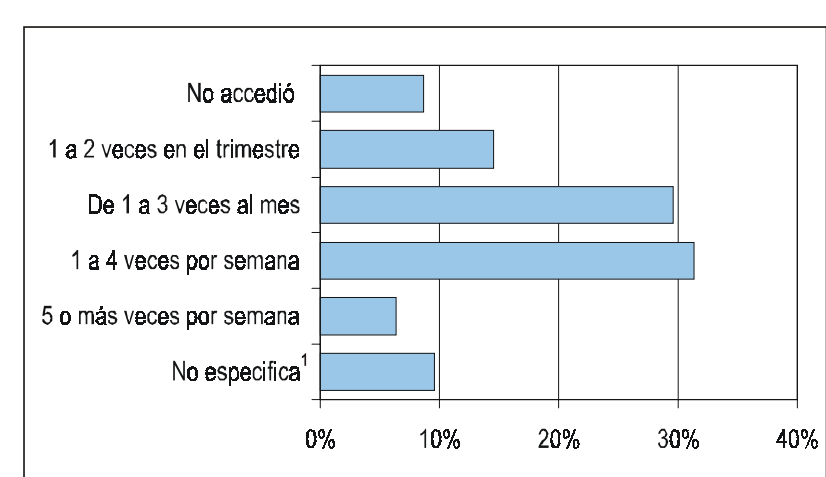

La frecuencia de acceso fue reportada como veces en el último trimestre, veces al mes, a la semana o al día (pregunta abierta). Las respuestas fueron aproximadas a una de las categorías ilustradas.

${ }^{1}$ Sujetos que manifestaron haber ingresado a la WuWven el último trimestre pero no especificaron con qué frecuencia.

Figura 2. Frecuencia de acceso a la WWW en el último trimestre.

este medio de comunicación (escribir un mail, entrar a un chat o navegar en la W W W), 23\% dejó la respuesta en blanco. Los valores de tiempo reportados por los que respondieron a esta pregunta sugieren un constante incremento en el porcentaje de estudiantes con algún tipo de experiencia en internet (Figura 4), de los cuales $21(12 \%)$ declararon haber tenido este primer contacto en el último trimestre. De manera similar, sólo 43,6\% de los estudiantes con internet en casa reportó el tiempo transcurrido desde que instalaron esta facilidad, de los cuales 9,7\% declaró haberlo hecho el último trimestre.

Los estudiantes definidos como hábiles en internet -según su capacidad reportada para realizar tareas básicas de navegación y mensajería (ver métodos)- se autoclasificaron en categorías de mayor experiencia en internet y en computadoras en comparación con los no hábiles ( $p<0,001$ en ambos casos), según las opciones expuestas en la Tabla 1. En este grupo, se encontró una mayor proporción de sujetos con conexión a internet en casa $(O R=4,56, I C$ $95 \%=2,28-9,14, p<0,001)$, que entre los no hábiles (Tabla 3). A simismo, los sujetos hábiles reportaron haber tenido su primer contacto con internet antes que los no hábiles, entrar a nave-

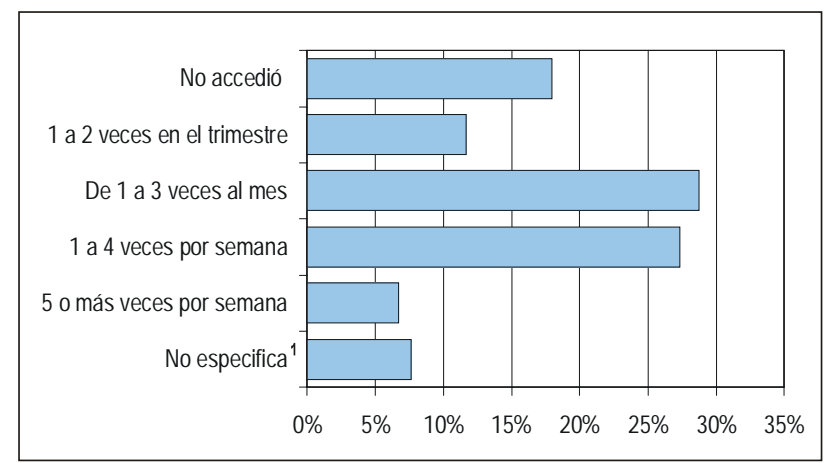

La frecuencia de acceso fue reportada como veces en el último trimestre, veces al mes, a la semana o al día (pregunta abierta), en las que el sujeto accedía a revisar su casilla de correo electrónico. Las respuestas fueron aproximadas a una de las categorías ilustradas.

${ }^{1}$ Sujetos que manifestaron haber accedido a su casilla de correo electrónico en el último trimestre, pero no especificaron con qué frecuencia.

Figura 3. Frecuencia de acceso al correo electrónico en el último trimestre.

gar a la WWW con mayor frecuencia, revisar su correo electrónico más frecuentemente y haber enviado y leído un mayor número de mensajes electrónicos en el último trimestre (Tabla 3). No se detectó diferencias significativas entre los sujetos hábiles y los no hábiles, en cuanto al año de estudios, la edad y el género.

\section{DISCUSIÓN}

Internet ha surgido como un medio de comunicación masivo, con el cual casi todos $(98,2 \%$ de los estudiantes en nuestra muestra) hemos tenido algún contacto. Sin embargo, es una herramienta relativamente compleja, que requiere una serie de habilidades para aprovecharla al máximo. Si bien en la literatura existen estudios que describen las características en países desarrollados del uso de internet por estudiantes (11-13) y profesionales de la salud (14-19), es poco lo que se conoce acerca de la situación en países en desarrollo. H asta nuestro conocimiento, éste es el primer reporte en la literatura que describe las características de una población de estudiantes de medicina latinoamericanos en cuanto sus habilidades, conocimientos y características del acceso a internet. 
Tabla 3. Diferencias entre los estudiantes hábiles y los no hábi les en la real ización de tareas en internet.

\begin{tabular}{|c|c|c|c|c|}
\hline & $n^{1}$ & Hábiles & No hábiles & $p$ \\
\hline $\mathrm{N}(\%)$ & 220 & $165(75 \%)$ & $55(25 \%)$ & - \\
\hline Edad & 217 & 21,44 & 21,91 & 0,36 \\
\hline Hombres:mujeres & 220 & $2,37: 1$ & $1,62: 1$ & 0,316 \\
\hline Identifica definición deinternet & 218 & $76,7 \%$ & $70,9 \%$ & 0,498 \\
\hline Internet en casa & 219 & $58,5 \%$ & $23,6 \%$ & $\varangle 0,001$ \\
\hline Tiempo desde primer contacto con internet (meses) ${ }^{2}$ & 171 & $12(24-36)$ & $6(1-24)$ & $\varangle 0,001$ \\
\hline Frecuencia de acceso a la WWW en el último tri mestre (veces $/ \mathrm{mes})^{2}$ & 194 & $12(4-24)$ & $4(0-4)$ & $\varangle 0,001$ \\
\hline Frecuencia de acceso al correo el ectrónico en el último trimestre (veces $/$ mes) ${ }^{2}$ & 201 & $9(4-30)$ & $1(0-4)$ & $\varangle 0,001$ \\
\hline Número de correos lé́dos en el úl timo trimestre (correos $/ \mathrm{mes})^{2}$ & 168 & $16(5-60)$ & $0(0-8)$ & $\varangle 0,001$ \\
\hline Número de correos enviados en el último trimestre (correos/mes)² & 202 & $8(3-30)$ & $0(0-2)$ & $\varangle 0,001$ \\
\hline
\end{tabular}

Los sujetos fueron clasificados arbitrariamente como hábiles o no hábiles, según un puntaje obteni do a partir de diez preguntas acerca de la capacidad para realizar tareas de mensajería y navegación en internet (ver materiales y métodos).

${ }^{1}$ Número de sujetos con una respuesta válida considerados en la estadística.

2 Mediana (percentil 25 - percentil 75).

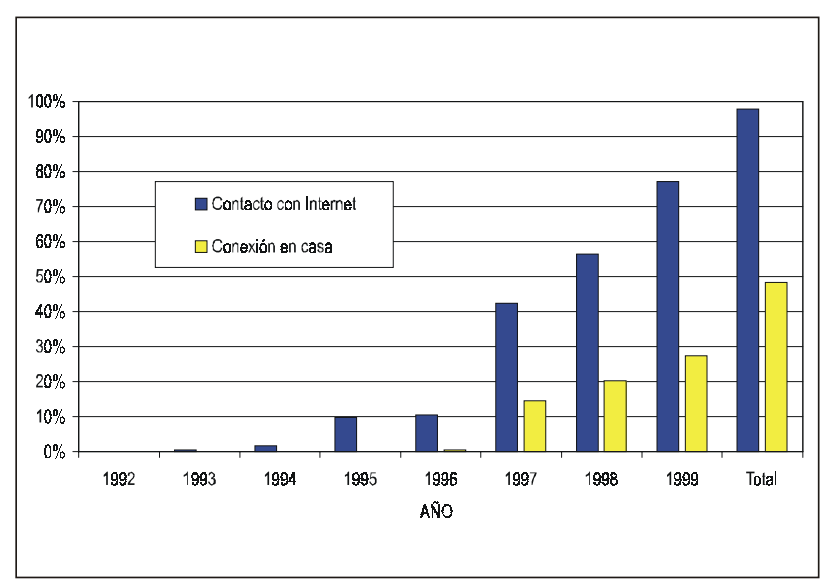

Las barras representan porcentajes acumulados del total de sujetos que respondieron la encuesta. El tiempo transcurrido desdeel primer contacto con internet (primera vez que se escribió un e-mail, se entró a un chat o se navegó en la WWWy y desde que se cuenta con una conexión a internet en casa fueron reportados como días, meses o años (pregunta abierta). Las respuestas fueron aproximadas a un determinado año.

1 Total de sujetos que reportaron haber teni do contacto con internet o tener conexión a internet en casa, incluyendo aquel los que no especificaron un valor de tiempo.

Figura 4. Año del primer contacto con internet y de la instalación del acceso a internet en casa.

En este estudio, la conexión a internet en casa fue el principal medio de acceso por los estudiantes de medicina, panorama similar al reportado para profesionales de la salud en países desarrollados $\left({ }^{14,15,19}\right)$. Si bien en nuestro medio las compañías proveedoras de internet están ofreciendo tarifas de conexión en casa cada vez más económicas y rápidas, todavía cerca de la mitad de estudiantes considera que el costo de conexión $(43,8 \%)$ y las fallas técnicas $(62,4 \%)$ representan limitantes al momento de buscar información médica en la red.

El tiempo transcurrido desde el primer contacto de los estudiantes con internet y desde la instalación del acceso a internet en casa (Figura 4), concuerda con la evolución de internet en el Perú $\left({ }^{10}\right)$, según la cual las primeras conexiones a la red por usuarios individuales y centros educativos se realizaron en 1993, mientras que recién en 1996 se inició un crecimiento exponencial del número de usuarios. Si bien la tasa de reporte de valores de tiempo en estas preguntas fue particularmente baja, los resultados obtenidos sugieren un constante incremento en el número de sujetos con acceso desde casa y un potencial para que continúe esta tendencia, considerando que cerca de la mitad de sujetos sin esta facilidad ya poseen una computadora.

El sistema de correo electrónico administrado por la universidad era utilizado sólo por $24,9 \%$ de los estudiantes encuestados, lo cual probablemente guarda relación con la gran va- 
riedad de cuentas de correo gratuitas disponibles en la W W W ${ }^{(20}$ ), la inclusión de cuentas de correo en el paquete de conexión a internet en casa y las distintas preferencias de los usuarios según sus necesidades y limitaciones. Existen otras al ternativas que deben ser exploradas para mantener comunicación vía correo electrónico entre los estudiantes, como por ejemplo la creación de listas de discusión ( ${ }^{21}$ ) o la redirección de los correos de un sistema único al sistema utilizado por cada estudiante (22).

A proximadamente 1 de 4 estudiantes no pudo identificar la definición correcta de internet (23), dentro de una serie de alternativas sencillas, Io cual suponemos refleja un desconocimiento de la manera cómo funciona este medio de comunicación. M ás aún, Ilama la atención que 53,8\% de estudiantes cree que la mayoría de información médica disponible en internet pueda ser usada como referencia confiable en un libro, revista o tesis (Tabla 2 ), cuando, dada la naturaleza anárquica de internet, sólo un pequeño porcentaje de la información publicada en la red es de una calidad aceptable para estos propósitos $(24-30)$.

Resulta particularmente preocupante que menos de la mitad de los alumnos haya reportado haber accedido a dos fuentes fundamentales de información médica en la red, como son las publicaciones confiables en texto completo $\left({ }^{31,32}\right)$ $(42,5 \%)$ y los consensos de tópicos médicos en internet $\left.(42,9 \%){ }^{33,34}\right)$. A ún si tuviesen conocimiento de estas direcciones, un número importante de estudiantes reportaron no saber realizar tareas básicas en internet (Tabla 1), tales como grabar una imagen de una página web en un disquete $(44,4 \%)$ o enviar un archivo por correo electrónico (39\%).

En el planeamiento de una estrategia de educación y promoción de internet en nuestro medio, resulta importante tomar en cuenta la heterogeneidad que existe entre los estudiantes de medicina en cuanto a sus habilidades y características de uso de internet, tal como se reporta en este estudio. En un esfuerzo por caracterizar a aquellos estudiantes poco hábiles, hemos identificado algunas variables que guardan relación estadística con un puntaje bajo en las preguntas acerca del dominio de herramientas de internet, como por ejemplo el no tener conexión en casa, un corto tiempo de experiencia con internet y un uso poco frecuente del correo electrónico y la W W W (T abla 3). Contrariamente a lo que se esperaría para otras habilidades o conocimientos inherentes a la práctica médica, el dominio de las herramientas básicas en internet no guarda relación con el año de estudios ni con la edad.

Teniendo como base los resultados obtenidos en esta encuesta, consideramos una prioridad mejorar la accesibilidad a internet entre los estudiantes de medicina, ya sea manteniendo un sistema eficiente de acceso gratuito desde el campus universitario y/o facilitando la adquisición de un acceso desde casa. A simismo, resaltamos la necesidad de implementar actividades educativas en internet, en las cual es el estudiante logre, entre otras cosas, 1) desarrollar un concepto acertado de internet en cuanto a la manera como funciona este medio y las características de la información disponible; 2) conocer y familiarizarse con las principales herramientas en internet para el estudiante de medicina, a manera de una lista de hipervínculos; y 3) ejercitarse en el manejo de archivos en internet, la instalación de software de la red y uso del correo electrónico.

La población estudiada en este trabajo no es sui generis. Por el contrario, comparte varias realidades con otros grupos estudiantiles en varias facul tades de medicina latinoamericanas, con quienes hemos podido intercambiar experiencias en el contexto de los congresos y cursos de la Sociedad Científica Peruana de Estudiantes de M edicina (SOCIPEM ) y la Federación L atinoamericana de Estudiantes de Medicina (FELSOCEM ). E speramos que este estudio haya logrado identificar problemas específicos, a los cuales deberán abocarse los esfuerzos por integrar a internet en la formación médica de esta población en particular y den luces acerca de los problemas predominantes en las muchas otras 
facultades de medicina del tercer mundo, las cuales comparten realidades similares a las de la población en este estudio.

\section{AGRADECIMIENTOS}

A la Sociedad Científica de Estudiantes de Medicina Cayetano Heredia - SOCEM CH.

\section{BIBLIOGRAFÍA}

1. Balteskard L, Rinde E. M edical diagnosis in the Internet age. The Lancet 1999; 354(Suppl 2): 2.

2. Weigle C, M arkovitz BP, Pon S. The Internet, the electronic medical record, the pediatric intensive care unit, and everything. Critical Care M ed 2001; 29(8)(Suppl 1): 166-76.

3. LaPorte RE, Akazawa S, Hellmonds P, Boosrom E, G amboa $C$, et al. Global public health and the information superhighway [E ditorial]. BM J 1994; 308: 1651-2.

4. Oreilly M. Broadcasting comes to the Internet. CM AJ 1999; 161(9): 1170.

5. W ootton R. Telemedicine. BMJ 2001;323:557-60.

6. Tucker J H, Busch C, Spatz A, W ells C, Brugal G. A n experimental inter-expert telepathology network using static imaging. J Clin Pathol 2001; 54(19): 752-7.

7. Prutkin J M. Cybermedical skills for the Internet age. JA M A 2001; 285(6): 808.

8. Le T, Stein M L. M edical education and the Internet: This changes everything. JA M A 2001; 285(6): 809.

9. The Internet and the developing world [N oticias]. BM J 1996; 316: 1111.

10. Díaz-A lbertini J. ¿Quo vadis, Internet? Scientia et Praxis, Lima 1997 enero-junio; №21, RB 1262 - V. 11. 510.

11. Obst 0 . Use of Internet resources by German medical professionals. Bull M ed Libr Assoc 1998; 86(4): 528-33.

12. Blumberg $P$, Sparks J. Tracing the evolution of critical evaluation skills in students' use of the Internet. Bull M ed Libr Assoc 1999; 87(2): 200-5.

13. Gibson KE, Silverberg M. A two-year experience teaching computer literacy to first-year medical students using skillbased cohorts. Bull M ed Libr A ssoc 2000; 88(2): 157-64.

14. Health on the Net Foundation. Surveys, evolution of Internet use for health purposes [Publicación en línea] U RL: http://www. hon. ch/Survey/ [acceso 19 diciembre 2001].

15. Lacher D, Nelson E, Bylsma W, Spena R. Computer use and needs of internists: a survey of the A merican College of Physicians-A merican Society of Internal Medicine. Proc A M IA Symp 2000; 453-6.

16. J adad AR, DPhil SC, Cocking LB, Lynda BA, Whelan $\mathrm{T}$, Browman $\mathrm{G}$. Internet use among physicians, nurses, and their patients [C orrespondencia]. JA M A 2001; 286(12); 1451-2.

17. Canadian Medical Association. 2000 CMA Physician resource questionnaire results [Publicación en línea]. URL: http://www. cma. ca/cmaj/vol-163/issue-5/prq/index. htm [acceso el 21 de diciembre 2001].

18. Kalsman M W, A costa DA. U se of the Internet as a medical resource by rural physicians. J Am Board Fam Pract 2000; 13(5): 349-52.

19. Chi-L um BI, Durkin RM . Physicians accessing the Internet: The PAI Project [Letter]. JA M A 1999; 282(7): 633-4.

20. Free email address directory [Publicación en línea]. U RL: http://www. emailaddresses. com/ [acceso 22 de diciembre 2001].

21. Harness e-mail: mailing lists [Publicación en línea]. U RL: http://www . learnthenet. com/english/html/24mlists. htm [acceso 22 de diciembre 2001].

22. Hayward $E$. The different types of free E mail [Publicación en línea]. URL: http://www.emailaddresses.com/ guide_types2.htm [acceso 22 de diciembre 2001].

23. All about the internet [Publicación en línea]. URL: http:// www.isoc.org/internet/ [acceso 22 de diciembre 2001].

24. Griffiths K, Christensen H. Quality of web based information on treatment of depression: cross sectional survey. BM J 2000; 321: 1511-5.

25. MCLeod SD. The quality of medical information on the Internet: A new public health concern [Editorial]. A rch Ophthalmol 1998; 116(12): 1663-5.

26. Eysenbach G, Diepgen TL. Towards quality management of medical information on the Internet: evaluation, labelling, and filtering of information. BMJ 1998; 317: 1496-500.

27. Coelho PC. The Internet: Increasing information, decreasing certainty. JAM A 1998; 280(16): 1454.

28. J adad AR, Gagliardi A. Rating health information on the Internet: Navigating to knowledge or to Babel? JA M A 1998; 279: 611-4.

29. Silberg WM, L umberg GD, M usacchio RA. A ssessing, controlling and assuring the quality of medical information on the Internet: Caveant lector et V iewer- L et the reader and Viewer Beware [E ditorial] JAM A 1997; 277: 1244-5.

30. Impicciatore P, Pandolfini C, Casella N, Bonati M. Reliability of health information for the public on the world wide web: systematic survey of advice on managing fever in children at home. BM J 1997; 214: 1875-81.

31. Free medical journals.com [Publicación en línea]. URL: http://www. freemedicaljournals. com [acceso 22 de diciembre 2001].

32. Medical Matrix [Publicación en línea]. URL: http:// www.medicalmatrix.com [acceso 22 de diciembre 2001].

33. Stewart A. Creating your own medical Internet library. CM AJ 1999; 161(9): 1155-60.

34. National Guideline Clearinghouse [Publicación en línea]. URL : http://www. guideline.gov [acceso 22 de diciembre 2001]. 